\title{
A test platform for cognitive delays: target tracking problem with multiple time-delayed feedback control
}

\author{
Qingbin Gao · Rudy Cepeda-Gomez · Nejat Olgac
}

Received: 13 September 2013 / Revised: 15 February 2014 / Accepted: 27 February 2014 / Published online: 20 March 2014

(C) Springer-Verlag Berlin Heidelberg 2014

\begin{abstract}
This paper studies a baseline 2-dimensional (2-D) target tracking problem from a new perspective. The dynamics entail two rationally independent time delays in an otherwise traditional feedback controller. A pursuer tries to track a moving target. When there are no time delays, capture is enabled using any stable control logic (for instance, $\mathrm{LQR}$ ). If there are different delays in the sensing of position and velocity of the target, however, the performance of the pursuit changes dramatically. We explore the stability of the dynamics for a broad range of delay compositions. An exhaustive and exact determination of those stable delay combinations is achieved using the recent cluster treatment of characteristic roots (CTCR) paradigm. It declares the complete stability outlook in the domain of the two delays. This method starts with the determination of all the potential stability switching curves in the domain of the delays. For this, a recent concept called the "spectral delay space (SDS)" is used. As a paradoxical outcome of this work, we show that the stability of the dynamics can be recovered by increasing the delays. The paper is accompanied by an interactive MATLAB-based game which is also available online.
\end{abstract}

Keywords CTCR $\cdot$ Multiple time delays $\cdot$ Stability · SDS $\cdot 2$-D target tracking

Q. Gao $\cdot$ R. Cepeda-Gomez $\cdot$ N. Olgac $(\bowtie)$

Department of Mechanical Engineering, University of Connecticut, Storrs, CT 06269, USA

e-mail: olgac@engr.uconn.edu

Q. Gao

e-mail: qig11001@engr.uconn.edu

R. Cepeda-Gomez

e-mail: rudycepeda@engr.uconn.edu

\section{Introduction}

The target tracking problem has many military and civilian applications, such as surveillance, torpedo guidance, robotic navigation. A recent survey [1] reviewed various mathematical models that have been developed in the past several decades for tracking a point target in the 2-D and 3-D space. In this paper, we study a 2-D point target tracking problem from an interesting perspective. A point pursuer tries to track a point target utilizing a certain real-time feedback control law, which enables the capture. When time delays are introduced into the control logic, however, due to the delayed sensory perception of the pursuer, the dynamics can show dramatic changes. The pursuer may or may not capture the target depending on these very critical parameters, the sensory (cognitive) time delays. An unconventional perspective is introduced, handling the influence of this feature on the system behavior.

The trajectory of the target is created in-situ by an outside user. This trajectory is obviously unknown to the pursuer a priori to the event. The overall dynamics is formulated as a linear time invariant (LTI) system with a full state feedback control which is influenced by two time delays. The position error vector and that of velocity error between the pursuer and the target are used in a conventional feedback control scheme. These error vectors are considered to be available to the controller with two time delays: one affecting the position error and the other is on the velocity error. The control logic is kept unchanged for all delay compositions. The focus in this effort is to determine the effects of the feedback delays on the control performance.

The trajectory tracking problem with time delayed feedback has received a great deal of attention in the control systems area. A recent paper [2] uses a Lyapunov-based adaptive control design to achieve the global stability of a general 
class of plants, under time-delayed state feedback. Reference [3] deals with a similar problem, using feed-forward adaptive control to compensate against the delay. LyapunovKrasovskii functional method is deployed to assure stability. A globally stable adaptive controller with a Smith-predictorlike structure for SISO input-delayed plants was analysed in [4]. Many control treatments encountered in literature resolve this problem by stabilizing the delayed dynamics using forecasting and state estimation methods and deploying the estimated states in the control law instead of their time-delayed forms. Similar treatments have been proven successful in many applications to the cost of numerical overhead and the drawbacks of uncertainty carried along with the adaptive procedures [5-8].

A remedial approach for the stability robustness analysis of this class of time-delayed dynamics is the cluster treatment of characteristic roots (CTCR) paradigm $[9,10]$. Some highlights and counter-intuitive findings from these works are listed as follows:

- The intuition "smaller the delay the better the control performance" is not always correct. Indeed, in some cases larger time delays may result in a better control. This feature chimes well with the realistic encounters in life that for some events a matured feedback signals results in better stabilization.

- Non-delayed controlled dynamics is designed to be stable. The same system may also be stable for non-zero delays which are represented by separated regions or "pockets" in the domain of the delays. The CTCR paradigm reveals these pockets exclusively [9]. The critical feature here is that once they are known, one can manipulate (prolong) the delays to restore stability within these regions.

- The stability feature of the controlled dynamics (i.e., the success of the capture) is decoupled from the actual trajectory followed by the target. This is a fundamental characteristic of the LTI dynamics, although in practice there are limitations such as the control sampling speed which can impose restrictions on the target dynamics for a successful pursuit.

We next discuss an important preliminary step which has to be implemented prior to the deployment of CTCR. It deals with the exact and exhaustive determination of all the potential stability switching curves in the delay space. This task is very complex. First, because the time-delayed systems possess infinitely many characteristic roots. Second, the dynamics at hand entails multiple rationally-independent delays. To handle this problem, we resort to a recently-introduced coordinate space here, which is called the Spectral Delay Space (SDS).

The contributions of this paper are threefold: (a) analytical derivations of the problem including the modelling and synthesis of the control logic;

(b) use of the SDS concept for the exact and exhaustive determination of potential stability switching curves and

(c) deployment of CTCR paradigm which determines the exact stability outlook of the system in the domain of the delays.

In parallel to this study a computer animation game is developed which is available online [11]. The game is interactively played by the users (who act as targets) validating some features of this time-delayed control scheme.

In the rest of the paper, bold face notation is used for vector quantities, bold capital letters for matrices and italic symbols for scalars. We refer to the right (and left) half open complex plane as $C^{+}\left(C^{-}\right)$, while $C^{0}$ is used to indicate the imaginary axis. Therefore, $C \equiv C^{+} \cup C^{-} \cup C^{0}$ represents the entire complex plane. The composition of the paper is as follows. In Sect. 2, we present the model of the dynamics. Section 3 treats the stability analysis, i.e., the CTCR paradigm deployment with SDS front-end. The case studies are in Sect. 4.

\section{System's model and control synthesis}

Both pursuer and target are considered to be point masses moving in a 2-D space. The position vectors of the pursuer and the target are denoted by $\mathbf{r}_{p}(t)=\left[x_{p}(t) y_{p}(t)\right]^{T}$ and $\mathbf{r}_{e}(t)=\left[x_{e}(t) y_{e}(t)\right]^{T}$, respectively. The pursuer is the only controlled agent, and its dynamics is given by $m \ddot{\mathbf{r}}_{p}(t)=$ $-c \dot{\mathbf{r}}_{p}(t)+\mathbf{u}_{p}(t)$, where $c$ is the viscous friction coefficient between the operating platform and the pursuer and $\mathbf{u}_{p}(t) \in$ $\mathfrak{R}^{2}$ is the control input. Considering the state vector $\mathbf{z}_{p}=$ $\left[x_{p} \dot{x}_{p} y_{p} \dot{y}_{p}\right]^{T}$, this decoupled dynamics can be represented in the state space as $\dot{\mathbf{z}}_{p}(t)=\mathbf{A}_{p} \mathbf{z}_{p}(t)+\mathbf{B u}(t)$, with the respective matrices:

$\mathbf{A}_{p}=\left[\begin{array}{cccc}0 & 1 & 0 & 0 \\ 0 & -\frac{c}{m} & 0 & 0 \\ 0 & 0 & 0 & 1 \\ 0 & 0 & 0 & -\frac{c}{m}\end{array}\right] \quad \mathbf{B}=\left[\begin{array}{cc}0 & 0 \\ \frac{1}{m} & 0 \\ 0 & 0 \\ 0 & \frac{1}{m}\end{array}\right]$

The target state, represented by $\mathbf{z}_{e}=\left[x_{e} \dot{x}_{e} y_{e} \dot{y}_{e}\right]^{T}$, follows a trajectory which is unknown a priori. In fact, in the computer game module, this trajectory is created by the mouse movements of the user. The target follows the trajectory of the mouse.

Since the pursuer is trying to capture the target, we define the error vector as:

$\mathbf{e}(t)=\mathbf{z}_{p}(t)-\mathbf{z}_{e}(t)$ 
and consider the following error dynamics:

$\dot{\mathbf{e}}(t)=\mathbf{A}_{p} \mathbf{e}(t)+\mathbf{B u}(t)$

For the control input $\mathbf{u}(t)$, we use full state feedback, $\mathbf{u}(t)=$ $-\mathbf{k e}(t)$ where

$\mathbf{k}=\left[\begin{array}{cccc}k_{11} & k_{12} & 0 & 0 \\ 0 & 0 & k_{23} & k_{24}\end{array}\right]$

The synthesized control logic is to be applied to the pursuer, transforming its dynamics into:

$\dot{\mathbf{z}}_{p}(t)=\mathbf{A}_{p} \mathbf{z}_{p}(t)-\mathbf{B k e}(t)$

The problem is now converted into a classical trajectory tracking treatment. At this point, we introduce a new perspective. Assume the pursuer is handicapped with some cognitive delays: a delay of $\tau_{1}$ seconds in the processing of position information and another delay of $\tau_{2}$ seconds in the velocity data. With these delays in place, the control logic in (4) cannot be applied, since the controller does not have access to the error vector at time $t$. It therefore uses the latest information available (with two delays) as if it were fresh.

We should stress that the full state feedback control law (k) has a direct effect on the stability of the system. When $\mathbf{k}=\mathbf{0}$ (i.e., uncontrolled system), the pursuer dynamics is marginally stable. When $\mathbf{k}$ is nonzero but small, the system is expected to remain stable even for some delays in the feedback lines. Such systems with small feedback gains have the 'delay independent stable' property [12]. That is, the control gain $\mathbf{k}$ is so feeble that the system remains always stable independent of the delays in the feedback lines. However, as $\mathbf{k}$ increases, the stability becomes delay dependent. Thus, the feedback control law $\mathbf{k}$ affects the stability property of the dynamics. However, one should notice that for the timedelayed system, there are no commonly accepted methodologies in the literature for the design of a stabilizing control law. Well-known procedures such as LQR are not feasible since the time-delayed system has infinite dimensionality. In this note, the control law is designed for the non-delayed dynamics for which the system has finite dimensionality and then delays are incorporated into the dynamics. Specifically, the feedback control law $\mathbf{k}$ is designed as a conventional optimal LQR controller for the non-delayed dynamics (4). Then we inject the feedback delays into the dynamics and it becomes:

$\dot{\mathbf{z}}_{p}(t)=\mathbf{A}_{p} \mathbf{z}_{p}(t)+\mathbf{B}_{1} \mathbf{e}\left(t-\tau_{1}\right)+\mathbf{B}_{2} \mathbf{e}\left(t-\tau_{2}\right)$ where the matrices affecting the delay terms are:

$\mathbf{B}_{1}=\left[\begin{array}{cccc}0 & 0 & 0 & 0 \\ -\frac{k_{11}}{m} & 0 & 0 & 0 \\ 0 & 0 & 0 & 0 \\ 0 & 0 & -\frac{k_{23}}{m} & 0\end{array}\right], \mathbf{B}_{2}=\left[\begin{array}{cccc}0 & 0 & 0 & 0 \\ 0 & -\frac{k_{12}}{m} & 0 & 0 \\ 0 & 0 & 0 & 0 \\ 0 & 0 & 0 & -\frac{k_{24}}{m}\end{array}\right]$

To assess the effect of the delays on the stability of this target tracking scenario, Eq. (5) is rewritten as:

$\dot{\mathbf{z}}_{p}(t)=\mathbf{A}_{p} \mathbf{z}_{p}(t)+\mathbf{B}_{1} \mathbf{z}_{p}\left(t-\tau_{1}\right)+\mathbf{B}_{2} \mathbf{z}_{p}\left(t-\tau_{2}\right)+\mathbf{F}$

With $\mathbf{F}=-\mathbf{B}_{1} \mathbf{z}_{e}\left(t-\tau_{1}\right)-\mathbf{B}_{2} \mathbf{z}_{e}\left(t-\tau_{2}\right)$ which is the exogenous stimulus coming from the user interface. The stability of this pursuer dynamics is defined by the homogeneous part of the equation. The respective characteristic equation becomes

$\operatorname{det}\left(s \mathbf{I}-\mathbf{A}_{p}-\mathbf{B}_{1} e^{-\tau_{1} s}-\mathbf{B}_{2} e^{-\tau_{2} s}\right)=0$

Declaration of the stability of (8) for all possible combinations of delays $\left(\tau_{1}, \tau_{2}\right) \in \mathfrak{R}^{2+}$ is not a trivial task. The difficulty is created, again, due to the infinite dimensionality which is introduced by the transcendental terms in (8). In the following section, we show how this task is efficiently addressed by using the CTCR paradigm.

\section{Stability analysis using CTCR paradigm and the sds domain}

The scalar characteristic Eq. (8), which dictates the dynamics of the pursuer, has the general formation of:

$$
\begin{aligned}
q\left(s, \tau_{1}, \tau_{2}\right)= & g_{1}(s)+g_{2}(s) e^{-\tau_{1} s}+g_{3}(s) e^{-\tau_{2} s} \\
& +g_{4}(s) e^{-2 \tau_{1} s}+g_{5}(s) e^{-2 \tau_{2} s} \\
& +g_{6}(s) e^{-\left(\tau_{1}+\tau_{2}\right) s}=0
\end{aligned}
$$

where $g_{k}(s)(k=1,2, \ldots, 6)$ are polynomials of s with real coefficients. This system is "retarded", meaning that $g_{1}(s)$ is the only term that contains the highest degree of $s$, which is 4 , and no delay is accompanying it. Equation (9) is a fourth order quasi-polynomial with commensuracy degree 2 (i.e., up to $2 \tau_{1}$ and $2 \tau_{2}$ terms appear) and cross-talk between the time delays (i.e., $\tau_{1}+\tau_{2}$ term is present). Solving for the stable regions of (9) is notoriously complex. A recent procedure which provides the exact stability map of this class of systems in the domain of the time delays is called the CTCR [9]. The main philosophy behind it is the "clustering" of all possible imaginary root crossings in the $\left(\tau_{1}, \tau_{2}\right) \in \mathfrak{R}^{2+}$ domain.

In brief, the procedure entails two essential steps: 
a) It starts with the exhaustive determination of the loci in the delay space $\left(\tau_{1}, \tau_{2}\right) \in \mathfrak{R}^{2+}$ where the system has imaginary characteristic roots. For this we follow a recent approach over a new domain which is called the SDS [13].

b) Assessing the stable and unstable regions in the $\left(\tau_{1}, \tau_{2}\right) \in$ $\mathfrak{R}^{2+}$ space which is now partitioned by infinitely many loci generated by (a).

Both tasks appear to be prohibitively involved due to infinite dimensionality of the problem. CTCR paradigm brings crucial discipline to this complexity and declares the desired stability outlook exhaustively and non-conservatively. In the following subsections we first describe the umbrella paradigm, CTCR, and its key propositions for the completeness of the discussion.

\subsection{Review of CTCR}

Definition 1 Kernel curves $\wp_{0}$ : The curves that consist of all the points $\left(\tau_{1}, \tau_{2}\right) \in \mathfrak{R}^{2+}$ exhaustively, which cause an imaginary root $s=\omega i, \omega \in \mathfrak{R}^{+}$and satisfy the constraint $0<\tau_{k} \omega<2 \pi(k=1,2)$ are called the kernel curves. The points on these curves contain the smallest delay compositions which correspond to all possible imaginary roots. By its definition, the kernel curves formation is unique for a given system (7).

Definition 2 Offspring curves $\wp$ : The curves obtained from the kernel curve by the following pointwise nonlinear transformation:

$\left\langle\tau_{1} \pm \frac{2 \pi}{\omega} j_{1}, \tau_{2} \pm \frac{2 \pi}{\omega} j_{2}\right\rangle, \quad j_{1}, j_{2}=0,1,2, \ldots$

are called the offspring curves. This definition simply utilizes the fact that any point on the kernel will result in $\infty^{2}$ (2 dimensional infinity) offspring.

Definition 3 Root Tendency, RT: The root tendency indicates the direction of transition of the imaginary root as only one of the delays, $\tau_{j}$, increases by $\varepsilon, 0<\varepsilon<<1$, while all the others remain constant:

$\left.\operatorname{RT}\right|_{s=\omega i} ^{\tau_{j}}=\operatorname{sgn}\left[\operatorname{Re}\left(\left.\frac{\partial s}{\partial \tau_{j}}\right|_{s=\omega i}\right)\right]$

There are two overarching propositions which form the pillars of the CTCR paradigm which are stated here without proof [9].

Proposition 1 Small number of kernel curves: The number of kernel curves is manageably small. To be specific for a LTITDS of state dimension $n$, the maximum possible number of kernel curves is $n^{2}$ [14].
Proposition 2 Invariant Root tendency property: Take an imaginary characteristic root, wi, caused by any one of the infinitely many grid points on the kernel and offspring curves in $\left(\tau_{1}, \tau_{2}\right) \in \Re^{2+}$ defined by the expression (10). The root tendency of these imaginary roots remains invariant from one 'offspring curve' to the other when one of the delays is kept constant. That is, the root tendency with respect to the variations of $\tau_{1}$ (or $\left.\tau_{2}\right)$ is invariant from the kernel to the corresponding offspring as $\tau_{2}\left(\right.$ or $\left.\tau_{1}\right)$ is fixed [15].

Spectral Delay Space (SDS): A new procedure is described in this segment for determining exhaustively the kernel (and offspring) curves. It is a recently formalized treatment [10, 13]. The procedure is developed on a new domain: SDS. This domain is defined by the coordinates $v_{j}=\tau_{j} \omega$ for every point $\left(\tau_{1}, \tau_{2}\right) \in \mathfrak{R}^{2+}$ on the kernel and the offspring curves. It represents a conditional mapping: if a delay set $\left(\tau_{1}, \tau_{2}\right)$ creates an imaginary root $\omega i$, (i.e., if the point is on the kernel or the offspring curves) then $\left(\tau_{1} \omega, \tau_{2} \omega\right)$ forms a point in the SDS. On the contrary, $\left(\tau_{1}, \tau_{2}\right)$ points that do not generate an imaginary root have no representation in the SDS since they are not important from the stability stand point.

The main advantage of SDS is that the representation of the kernel curve in this domain, denoted as $\wp_{0}^{S D S}$ and called the building curve, is confined into a square of edge length $2 \pi$. Then, it is only necessary to explore a finite domain to find the representation of the building curves in the SDS. This finite domain is known as the building block (BB), i.e., $2 \pi \times 2 \pi$ squares, as per (10). The curves which correspond to the offspring in SDS are called the reflection curves. Another advantage of these coordinates is that the transition from the building to the reflection curves is achieved simply by stacking the copies of the BB as opposed to using the pointwise nonlinear transformation (10) in the space of the delays $\left(\tau_{1}, \tau_{2}\right)$. This prevents the appearance of an undesirable shape distortion. There are several other intriguing properties of the SDS and BB concepts [10]. Here, we introduce a few of them without proof.

Property 1 Kernel isolation property. The BB contains no trace of reflection curves in the SDS.

Property 2 The building curves end on the surface of the BB or close onto themselves inside the $\mathrm{BB}$.

Property 3 Symmetricity property of building curves. The point $(\pi, \pi)$ in SDS is the centre of symmetry of the BB as well as the building curves.

\subsection{Stability analysis in the SDS domain}

With these definitions and propositions, we now return to the earlier mentioned step (a) of CTCR method. It is the exhaustive determination of all the imaginary roots, $s=\omega i$, 
for the characteristic equation in (8) within the semi-infinite quadrant of $\left(\tau_{1}, \tau_{2}\right) \in \mathfrak{R}^{2+}$. We follow the mathematical procedure described by reference [13] which evaluates the building curves. Accordingly, in (9), the exponential terms are replaced by:

$e^{-\tau_{k} \omega i}=\cos \left(v_{k}\right)-i \sin \left(v_{k}\right), \quad v_{k}=\tau_{k} \omega, \quad k=1,2$

and the sine and cosine functions are expressed in terms of half-angle tangent function:

$\cos \left(v_{k}\right)=\frac{1-z_{k}^{2}}{1+z_{k}^{2}}, \quad \sin \left(v_{k}\right)=\frac{2 z_{k}}{1+z_{k}^{2}}, \quad z_{k}=\tan \left(v_{k} / 2\right)$

Equation (9) can now be written as a polynomial in $\omega$ with complex coefficients $c_{k}$ parameterized in $z_{1}$ and $z_{2}$ :

$q\left(\omega, z_{1}, z_{2}\right)=\sum_{k=0}^{4} c_{k}\left(z_{1}, z_{2}\right)(\omega i)^{k}=0$

If there is a solution $\omega \in \mathfrak{R}^{+}$to (14), both its real and imaginary parts must be zero simultaneously:

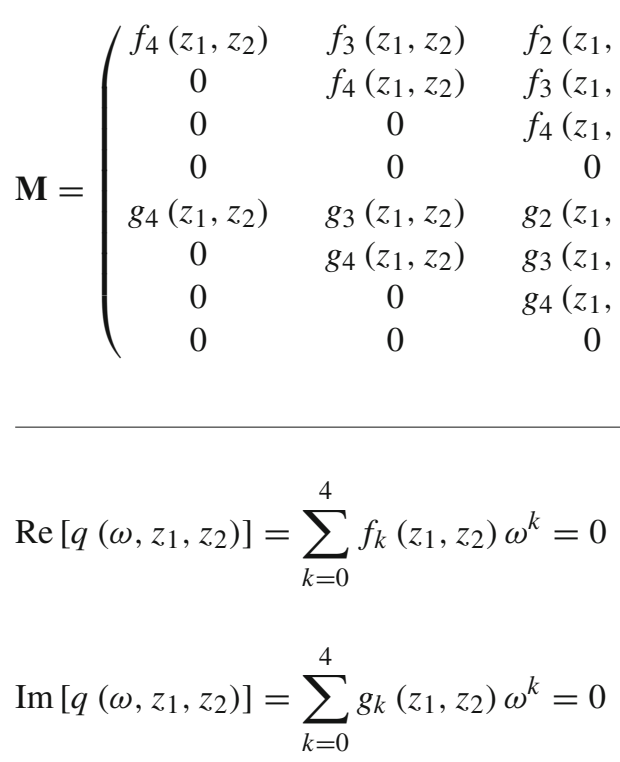

The condition for (15a) and (15b) to have a common root $\omega$, is that Sylvester's resultant matrix, which is defined in (17), must be singular. This results in the following expression in terms of $z_{1}$ and $z_{2}$ :

$\operatorname{det}(\mathbf{M})=F\left(z_{1}, z_{2}\right)=F\left(\tan \left(v_{1} / 2\right), \tan \left(v_{2} / 2\right)\right)=0$ which constitutes a closed form description of the kernel curves in the $\operatorname{SDS}\left(v_{1}, v_{2}\right)$, i.e., the building curves. To obtain its graphical depiction, one of the parameters, say $v_{2}$, can be scanned in the range of $[0,2 \pi]$ and the corresponding $v_{1}$ values are calculated again in $[0,2 \pi]$. Notice that every point $\left(v_{1}, v_{2}\right)$ on these curves brings an imaginary characteristic root at $\pm \omega i$ which can be evaluated from (15a) or (15b), noting that they share the same $\omega$ root. That is, we create a continuous sequence of $\left(v_{1}, v_{2}, \omega\right)$ sets all along the kernel curves. We then back transform from the $\left(v_{1}, v_{2}\right)$ domain of SDS to the $\left(\tau_{1}, \tau_{2}\right)$ delay space, using the inverse transformation of (12) with the appropriate $\omega$ values. This generates the kernel and offspring curves exhaustively, and it is the end of step (a) listed above.

The kernel and offspring curves divide the $\left(\tau_{1}, \tau_{2}\right)$ domains in regions of possible stability and instability. To determine the stability nature of these regions we start from the non-delayed system (i.e., $\tau_{1}=\tau_{2}=0$ ). Obviously, system (3) starts as stable for the non-delayed case, by the very design of the controller.

The root tendency invariance property is deployed from one region to the other, resulting in the complete and exact stability outlook of the system, which completes the step (b) mentioned at the beginning of the section.

\subsection{Steady-state trajectory tracking error}

In this subsection we investigate the performance from the perspective of steady-state tracking error, in response to different evader actions (reference inputs). Ideally it should be zero for a perfect tracking.

We start by replacing (2) into (5):

$$
\begin{aligned}
\dot{\mathbf{e}}(t)+\dot{\mathbf{z}}_{e}(t)= & \mathbf{A}_{p} \mathbf{e}(t)+\mathbf{A}_{p} \mathbf{z}_{e}(t) \\
& +\mathbf{B}_{1} \mathbf{e}\left(t-\tau_{1}\right)+\mathbf{B}_{2} \mathbf{e}\left(t-\tau_{2}\right)
\end{aligned}
$$

Furthermore, taking advantage of the decoupled dynamics in the two spatial coordinates, we look at the error dynamics in 
$x$ direction only. The treatment would be similar for $y$ :

$\ddot{e}_{x}+\ddot{x}_{e}=-\frac{c}{m} \dot{e}_{x}-\frac{c}{m} \dot{x}_{e}-k_{11} e_{x}\left(t-\tau_{1}\right)-k_{12} \dot{e}_{x}\left(t-\tau_{2}\right)$

Laplace transformation of this equation, assuming zero initial conditions, yields:

$\frac{E_{x}(s)}{X_{e}(s)}=\frac{-s\left(s+\frac{c}{m}\right)}{s^{2}+\frac{c}{m} s+k_{11} e^{-\tau_{1} s}+k_{12} s e^{-\tau_{2} s}}$

It can be shown that the denominator of (20) is one of the two factors of the characteristic Eq. (8). Similarly, the denominator of the error in y direction, $E_{y}(s)$, is the other factor, i.e., $s^{2}+\frac{c}{m} s+k_{23} e^{-\tau_{1} s}+k_{24} s e^{-\tau_{2} s}$.

For the stable delay combinations where all the poles of $s E_{x}(s)$ lie in the left half complex plane one can apply the Final Value Theorem [16]. It is trivial to show that if the input is a step function, $x_{e}(t)=1$ (i.e., the target jumps from one location to the other) the tracking error becomes zero, for a unit ramp action (evader moving with constant speed) and $x_{e}(t)=t$ we obtain a steady-state position error:

$e_{x}(\infty)=\lim _{s \rightarrow 0} s E_{x}(s)=-\frac{c}{m k_{11}}$

The corresponding velocity error in $\mathrm{x}$ direction is:

$\dot{e}_{x}(\infty)=\lim _{s \rightarrow 0} s^{2} E_{x}(s)=0$

For a constant acceleration input on the other hand, $x_{e}(t)=$ $t^{2}$, the steady-state tracking error becomes infinity.

In summary, a stable pursuer with the given full-state control logic is able to track a target which moves in step function jumps to a new position. It can also track a target moving at a constant velocity with an adjustable finite position error but will not be able to track one moving with a constant acceleration.

It can be seen from (21) that the steady state position error in $\mathrm{x}$ direction to a unit ramp input is a function of the viscous friction coefficient, $c$ (between the operating platform and the pursuer), the mass $m$ and $k_{11}$ (a component of the gain matrix k). It obviously, does not depend on the time delays. Since for a given pursuer $c$ and $m$ are fixed, one can reduce the tracking error in response to the unit ramp target motion by enlarging the absolute value of $k_{11}$ the control gain. This adjustment can also be achieved by properly selecting the weighting matrices $\mathbf{Q}$ and $\mathbf{R}$ at the very beginning. A numerical example in the following section illustrates these features.

\section{Example case and hands-on computer game}

\subsection{Stability assessment}

For our model, the pursuer parameters are selected as $m=1$ and $c=3$. The $\mathbf{Q}$ and $\mathbf{R}$ matrices used in the synthesis of the controller are:

$\mathbf{Q}=\left[\begin{array}{cccc}20 & 0 & 0 & 0 \\ 0 & 10 & 0 & 0 \\ 0 & 0 & 20 & 0 \\ 0 & 0 & 0 & 10\end{array}\right], \mathbf{R}=\left[\begin{array}{ll}2 & 0 \\ 0 & 4\end{array}\right]$

which lead to the LQR feedback gain matrix:

$\mathbf{k}=\left[\begin{array}{cccc}3.1623 & 1.5083 & 0 & 0 \\ 0 & 0 & 2.2361 & 0.9965\end{array}\right]$

With these numerical values, we perform the stability assessment procedures described in the previous Section. The corresponding characteristic equation is in the generic form of (9).

The building and reflection curves in the SDS (red and blue, respectively) are shown in Fig. 1. Transforming them back to the $\left(\tau_{1}, \tau_{2}\right)$ domain, the stability outlook for the system is obtained, as in Fig. 2. The shaded region represents the delay combinations which guarantee stability for the system and the eventual capture of the target by the pursuer. The points outside that region render the system unstable, and the target will escape from the pursuer. Two arbitrary points

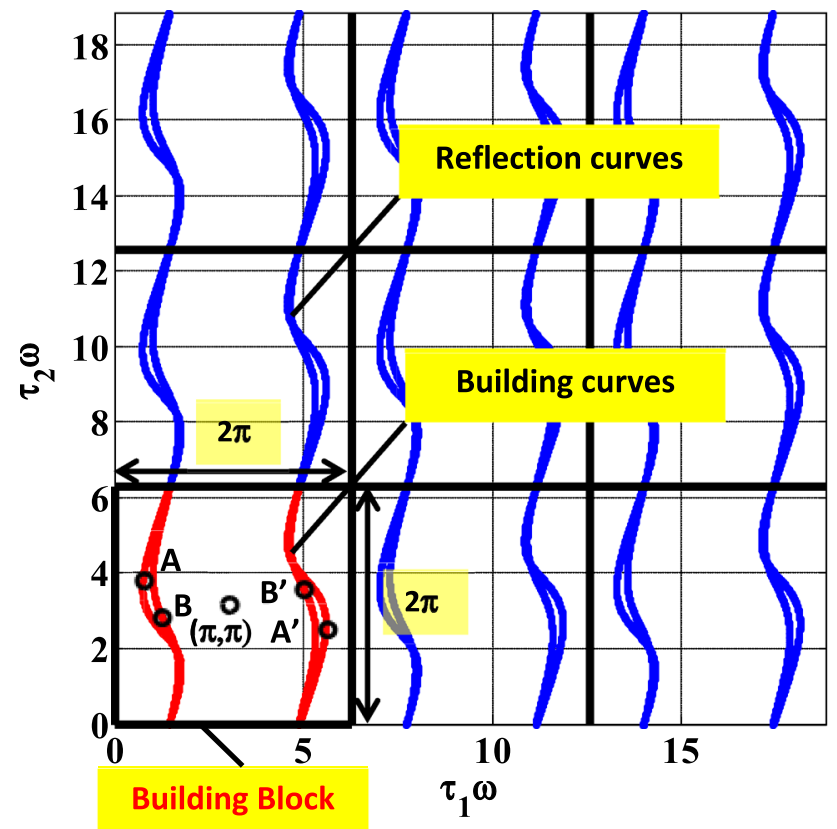

Fig. 1 Spectral Delay Space (SDS) representation of the stability switching curves. (Color figure online) 


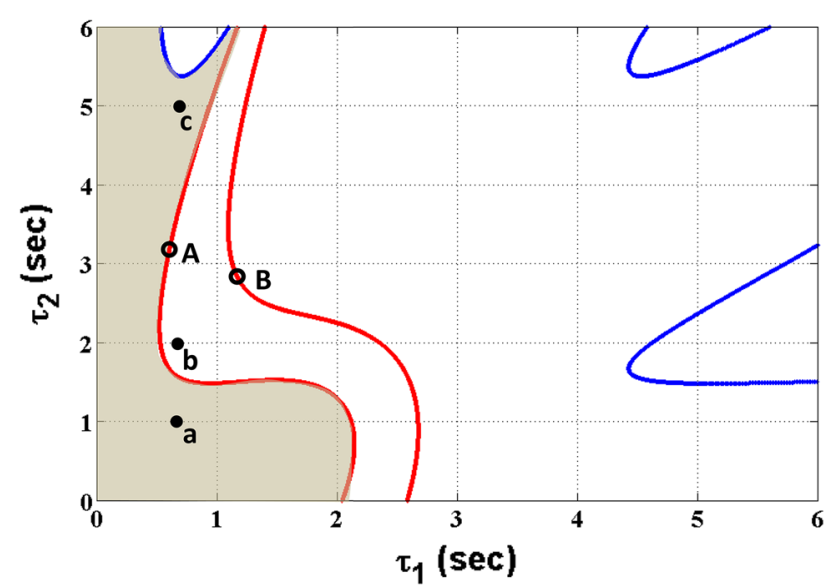

Fig. 2 Stability map in $\tau_{1}-\tau_{2}$ space with viscous friction coefficient $c=3$. Shaded region is stable. Red curves are kernel curves and blue ones are offspring curves. (Color figure online)

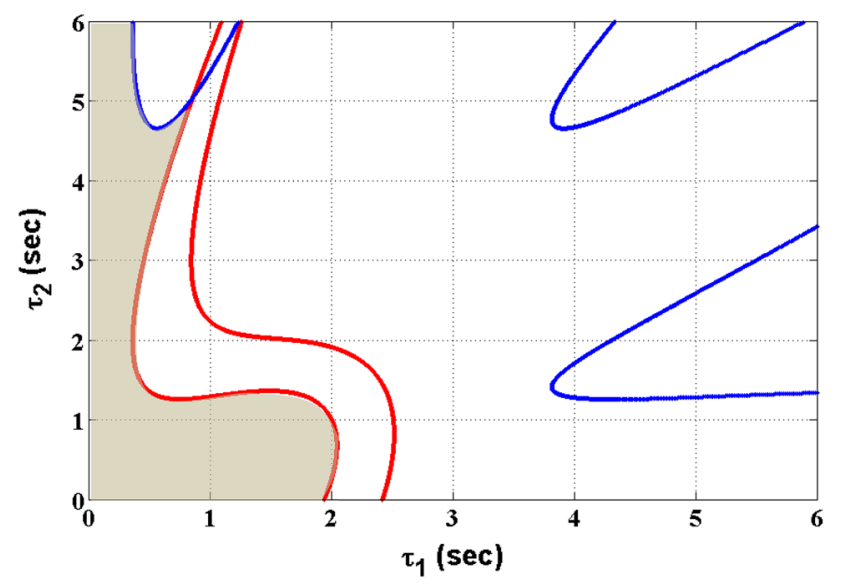

Fig. 3 Stability map in $\tau_{1}-\tau_{2}$ space with viscous friction coefficient $c=3 \times 90 \%$. Shaded region is stable. Red curves are kernel curves and blue ones are offspring curves. (Color figure online)

on the kernel, $A\left(\tau_{1 A}=0.60, \tau_{2 A}=3.12, \omega_{A}=1.19\right)$ and $B\left(\tau_{1 B}=1.21, \tau_{2 B}=2.73, \omega_{B}=0.60\right)$ are taken in Fig. 2 just to display their correspondence in Fig. 1 on the building curves. One can also see the symmetry property (see Property 3 of SDS and BB concepts) between $A(0.71,3.71)$ and $A^{\prime}(5.57,2.57)$ with the common imaginary root, $\omega_{A}$. The same argument is valid between $B(1.24,2.81)$ and $B^{\prime}(5.04,3.47)$. Notice that $A$ and $A^{\prime}$ (also $B$ and $B^{\prime}$ ) coincide in the delay space shown in Fig. 2.

To study the damping effect (viscous friction coefficient) on the pursuer and the stability of the delayed dynamics, we produce the stability maps separately for two cases: one with $10 \%$ decreased viscous friction (Fig. 3) and the other with $10 \%$ increased friction (Fig. 4). One can see that the area of the stable region in Fig. 3 decreases compared with that in Fig. 2 and the area of the stable region in Fig. 4 is increased. The intuitive explanation for this is that, since the norm of

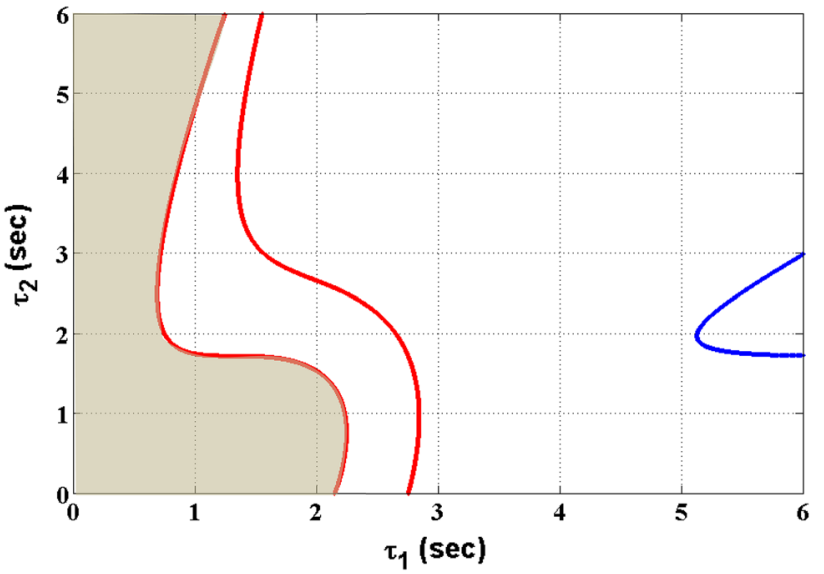

Fig. 4 Stability map in $\tau_{1}-\tau_{2}$ space with viscous friction coefficient $c=3 \times 110 \%$. Shaded region is stable. Red curves are kernel curves and blue ones are offspring curves. (Color figure online)

the matrix $\mathbf{A}_{p}$ is decreased with the reduced viscous friction coefficient $c$, the delays have a larger effect on the stability of the system given the fixed control law. Thus, the stability becomes more delay sensitive and the stable region shrinks. Figure 5 shows the impulse responses of the system (7) for three arbitrarily selected delay combinations corresponding to points $\mathbf{a}, \mathbf{b}$ and $\mathbf{c}$ in Fig. 2.

Notice that by increasing the time delay $\tau_{2}$ while keeping $\tau_{1}$ fixed from point $\mathbf{b}$ to $\mathbf{c}$ the system recovers stability. The implications from this observation are clear: if the delays which are inherent to the sensing process render the system unstable, the controller can artificially increase these delays (for instance by introducing additional "hold buffers" in the feedback line) to recover the stability. This is the reflection of a recent concept, delay scheduling [17,18]. The stability is regained simply by manipulating the delays, without changing the existing control law. Such a strategy is applicable to practical systems with delayed feedback control. However, the implementation of delay scheduling requires the exact knowledge of the stability outlook of the given system in the delay space. The CTCR paradigm serves exactly this purpose.

The settling time of the system, which is the time required by the pursuer to capture the target is also an interesting feature to look at. It depends on the rightmost characteristic roots (dominant roots) of the system. They are declared by the characteristic equation in the form of (9). The roots of this quasi-polynomial can only be calculated by some numerical approximation codes [19-21]. Here, we deploy the QPmR (Quasi-Polynomial, mapping-based Root finding) algorithm [19]. For each point $\left(\tau_{1}, \tau_{2}\right)$ on a fine grid the dominant roots and their real parts, $\operatorname{Re}\left(s_{d o m}\right)$, are determined. The corresponding variations are displayed in Fig. 6.

Some highlight observations over these figures are as follows: 
Fig. 5 Impulse response of the pursuer's dynamics. From top to bottom, $\tau_{1}=0.7 \mathrm{~s}, \tau_{2}=1 \mathrm{~s}$, point (a) in Fig. 2,

$\tau_{1}=0.7 \mathrm{~s}, \tau_{2}=2 \mathrm{~s}$, point $(\mathbf{b})$ in Fig. 2, and $\tau_{1}=0.7 \mathrm{~s}, \tau_{2}=5 \mathrm{~s}$, point (c) in Fig. 2. (Color figure online)
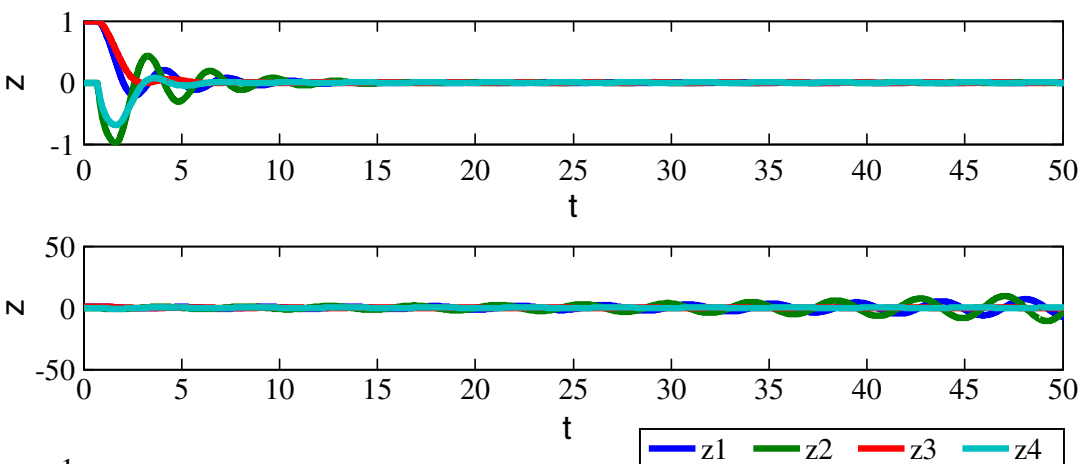

$N$

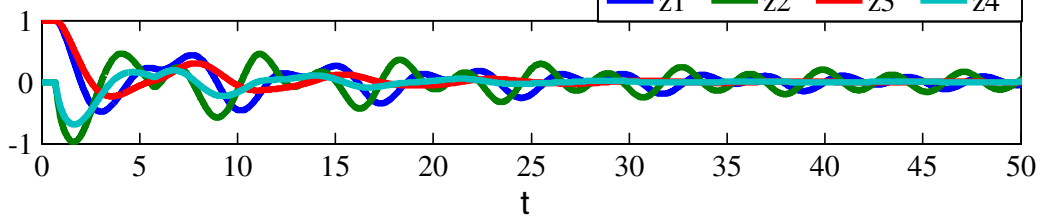

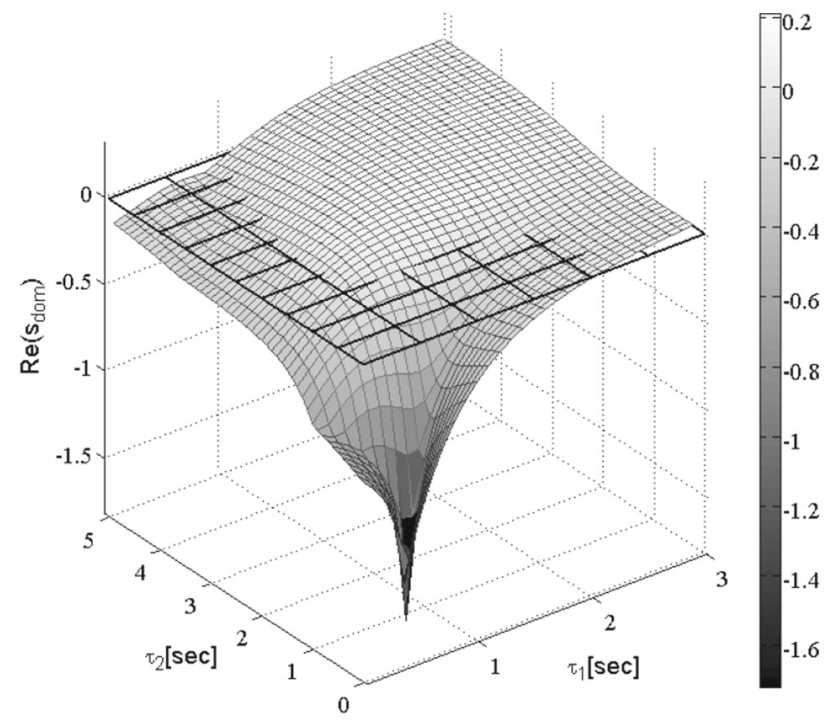

Fig. 6 Real part of the dominant root of the system

(i) The stable zones (shaded regions) of Fig. 2 nicely coincide with the regions where $\operatorname{Re}\left(s_{d o m}\right)<0$ in Fig. 6, as well as the unstable zones.

(ii) The time constant expected for capturing the target is $-1 / \operatorname{Re}\left(s_{d o m}\right)$, i.e., in example case a of Fig. 2 exactly agrees with the location of the dominant root, $s_{d o m}$. For this particular point, $\operatorname{Re}\left(s_{d o m}\right)$ is -0.28 which corresponds to a time constant of $3.6 \mathrm{~s}$, and a settling time of approximately $14.4 \mathrm{~s}$, as visible in simulations in Fig. 5 .

(iii) Paradoxically, the shortest settling time is achieved at $\tau_{1}=0.61 s$ and $\tau_{2}=0.51 s$ with $\operatorname{Re}\left(s_{d o m}\right)$ being -1.7193 instead of $\tau_{1}=\tau_{2}=0$ with $\operatorname{Re}\left(s_{\text {dom }}\right)$ being -0.6768 . Obviously, the former set of time delays makes a better choice from the point of view of capturing speed, although the delays are larger. This is a counter-intuitive observation and it is one of the most practical advantage of delay scheduling concept $[17,18]$. It implies that we can artificially increase the delay to restore stability and also to create a better disturbance rejection speed.

\subsection{Hands-on computer game}

An interactive computer graphics demo is implemented in Matlab R2010a on this theme called the "Homicidal Chauffeur Game". Figure 7 shows the main block diagram. The block marked in blue is the motion controller for the target, which handles the conversion of the movements of the mouse to the target's movements.

The blocks marked in red describe the dynamics of the chauffeur (pursuer) and the green block includes an animation (display) function. The complete coding for this system is available for download at the ALARM laboratory website [11].

\section{Conclusions}

This paper presents a classic 2-D target tracking problem under a new perspective. The objective of the mission is the capture of a target by a pursuer, which has delayed cognitive capabilities. One of the delays affects the position sensing and the other one is on the velocity. An optimal LQR controller is designed to guarantee capture for the non-delayed case. When the delays are present, however, the performance of the system degrades and the dynamics may become unstable.

The CTCR paradigm is used to assess the stability of the system and obtain the exact stability outlook in the domain of the delays. We show that it is possible to declare exactly which delay combinations result in a successful game (i.e., capture). The deployment of CTCR requires all the delay compositions which render imaginary characteristic roots. To obtain this knowledge we use a new surrogate space, called 


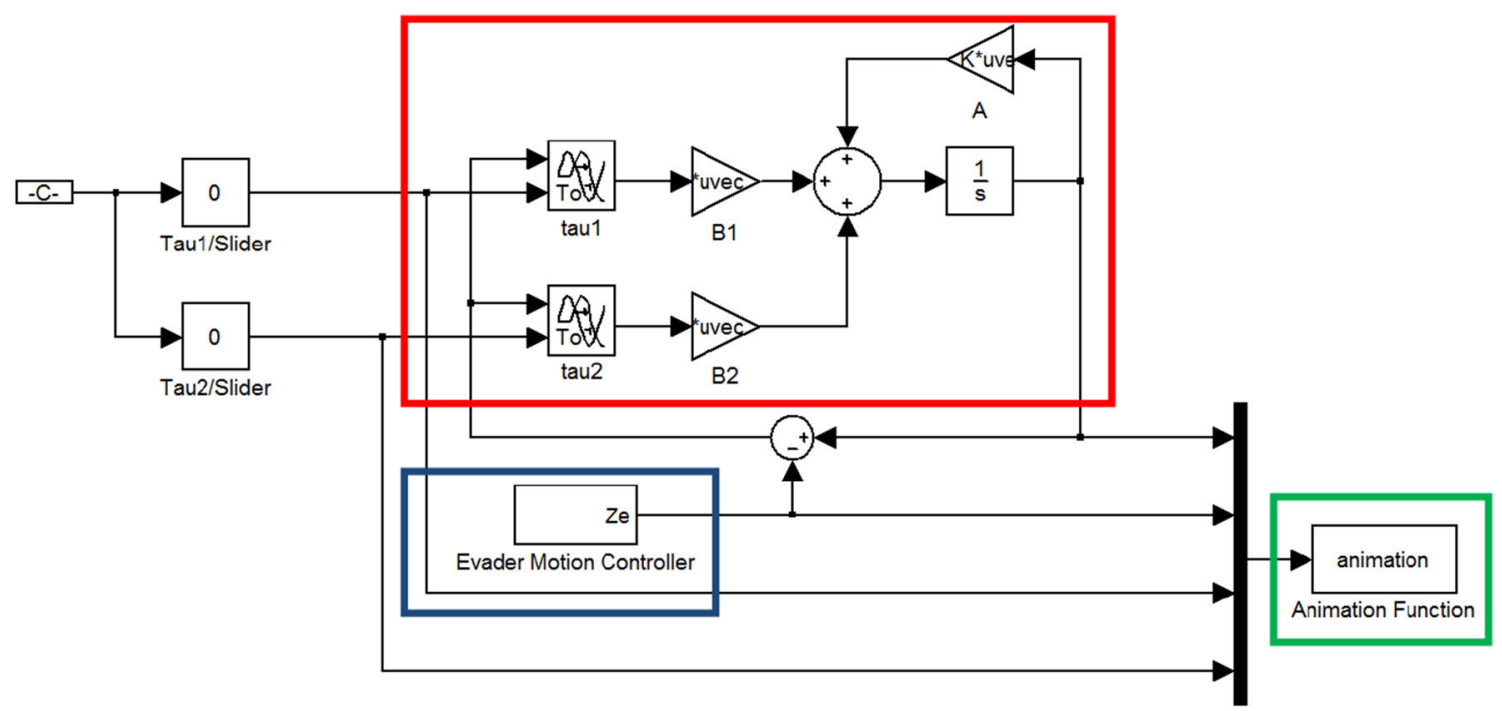

Fig. 7 Simulink block diagram of the interactive hands on computer game. (Color figure online)

the Spectral Delay Space. Ultimately this exact stability outlook of the system enables a new capability called the Delay Scheduling.

The stability results are validated by means of a Simulinkbased interactive demo, which is available for download at the ALARM website of the authors [11].

Acknowledgments An earlier version of this effort was performed at different stages by Ali Fuat Ergenc, Rifat Sipahi and Emre Cavdaroglu. Both the dynamics and the respective simulation codes are now completely revised, although the underlying scheme of the target tracking remains the same. We, therefore, wish to acknowledge the earlier efforts of the mentioned researchers at ALARM Lab in UCONN.

\section{References}

1. Li XR, Jilkov VP (2003) Survey of maneuvering target tracking. Part I: dynamics models. IEEE Trans Aerosp Electron Syst 39(4):1333-1364

2. Bresch-Pietri D, Krstic M (2009) Adaptive trajectory tracking despite unknown input delay and plant parameters. Automatica 45:2074-2081

3. Mirkin B, Gutman P (2003) Output-feedback model reference adaptive control for continuous state delay systems. J Dyn Syst Meas Control Trans. ASME 125:257-261

4. Ortega R, Lozano R (1988) Globally stable adaptive controller for systems with delay. Int J Control 47(1):17-23

5. Koivo AJ, Repperger DW (1973) On a game problem involving systems with time delay. IEEE Trans Autom Control 18(2):149152

6. Kang BS, Kim SH, Kwak YK, Smith CC (1999) Robust tracking control of a direct drive robot. ASME J Dyn Syst Technol 121:261269

7. Ichikawa K (1986) Adaptive control of delay system. Int J Control 41:1653-1659

8. Niculescu SI, Annaswamy AM (2000) A simple adaptive controller for positive-real systems with time-delay. In: Proceedings of the American control conference, Chicago, IL:3666-3670.
9. Olgac N, Sipahi R (2005) Complete stability robustness of thirdorder LTI multiple time-delayed systems. Automatica 41(8):14131422

10. Fazelinia H, Olgac N, Sipahi R (2007) Stability robustness analysis of multiple time-delayed systems using building block concept. IEEE Trans Autom Control 52(5):1413-1422

11. Cepeda-Gomez R, Gao QB (2012) Homicidal chauffeur problem simulation with delayed feedback. Alarm Laboratory, University of Connecticut. http://engr.uconn.edu/alarm/research?id=62 24 January 2012

12. Gu K, Kharitonov VL, Chen J (2002) Stability of time-delayed systems. Birkhauser, Boston

13. Fazelinia H (2007) A novel stability analysis of systems with multiple delays and its application to high speed milling chatter. PhD Dissertation, University of Connecticut.

14. Ergenc AF, Olgac N, Fazelinia H (2007) Extended Kronecker summation for cluster treatment of LTI systems with multiple delays. SIAM J Control Optim 46(1):143-155

15. Olgac N, Sipahi R (2002) An exact method for the stability analysis of time delayed LTI systems. IEEE Trans Autom Control 47(5):793-797

16. Ogata K (2004) System Dynamics, 4th edn. Prentice-Hall Inc, Upper Saddel River

17. Olgac N, Sipahi R, Ergenc A (2007) 'Delay scheduling', an unconventional use of time delay for trajectory tracking. Mechatronics 17(4):199-206

18. Olgac N, Cavdaroglu M (2011) Full state feedback control design with delay scheduling for cart-and-pendulum dynamics. Mechatronics 21(1):38-47

19. Vyhdilal T, Zitek P (2009) Mapping based algorithm for large scale computation of quasi-polynomial zeros. IEEE Trans Autom Control 54(1):171-177

20. Breda D, Maset S, Vermiglio AR (2006) Pseudospectral differencing methods for characteristic roots of delay differential equations. SIAM J Sci Comput 27(2):482-495

21. Engelborghs K, Roose D (2002) On stability of LMS methods and characteristic roots of delay differential equations. SIAM J Numer Anal 40(2):629-650 